\title{
RECONSTRUÇÃO DE DENTE ANTERIOR EM RESINA COMPOSTA UTILIZANDO GUIA DE SILICONE
}

\section{Reconstruction of anterior teeth in composite resin using silicon guide}

\author{
Lizete Karla Filgueiras de Souza ${ }^{1,2}$, Samara Pinho Assi ${ }^{1,2}$, Raphael Carvalho e Silva ${ }^{2}$, \\ Taís Sanae Fernandes Fukunaga ${ }^{2}$, Ana Emília Farias Pontes ${ }^{3}$, Alex Tadeu Martins ${ }^{1}$ \\ ${ }^{1}$ Curso de Mestrado em Ciências Odontológicas, Centro Universitário da Fundação Educacional de Barretos \\ - UNIFEB. \\ ${ }^{2}$ Faculdade de Odontologia do Amazonas - IAES. \\ ${ }^{3}$ Universidade Federal de Juiz de Fora campus Governador Valadares - UFJF/GV.
}

\begin{abstract}
RESUMO
O restabelecimento do sorriso harmônico é um desafio na odontologia. Nos casos de fraturas em dentes anteriores, a técnica restauradora direta em resina composta é uma abordagem conservadora que possibilita a reconstrução e preservação do remanescente dentário, recriando uma estética natural e função morfológica. O objetivo desse trabalho foi apresentar um caso clínico em que a reconstrução de uma fratura coronária do elemento 11 no terço médio foi realizada por meio da restauração direta em resina composta nanoparticulada com auxílio de guia de silicone, demonstrando ser uma técnica com resultado conservador, estético, satisfatório e de menor custo ao paciente.
\end{abstract}

Palavras-Chave: Resina Composta. Guia de Silicone. Reconstrução Dentária.

\begin{abstract}
The restoration of a satisfactory smile is a challenge in dentistry, as anterior tooth fractures are increasingly frequent. Thus, direct restorative composite resin technique became more efficient in providing a conservative approach restorative procedures, enabling the reconstruction and preservation of the natural teeth remnant recreating their morphological appearance, and their function. Therefore, the aim of this study was to present a case through the reconstruction of a coronal fracture of the element 11 in the middle third through direct restoration in composite nanoparticle with aid of silicon guide. Proving being a technique with conservative result, aesthetic, satisfying, and lower cost to the patient.
\end{abstract}

Keywords: Composite Resin. Guide of Silicon. Dental Reconstruction.

\footnotetext{
Autor para Correspondência: Lizete Karla Filgueiras de Souza.

Rua 5, n 134, Conj. Castelo Branco, Bairro Parque Dez - CEP 69055-230. Manaus - AM

Email: lizetefilgueiras@hotmail.com

Telefone: (92) 3236-2109 / (92) 98160-4044
}

Recebido em: 16/04/15

Aceito para publicação em: 01/03/2016

https://doi.org/10.4322/1980-0029.062015 


\section{INTRODUÇÃO}

As fraturas dentárias frequentemente acometem os incisivos superiores, o que pode ser explicado pela sua proeminência no arco. Nestes casos, o tratamento pode ser realizado por meio de reparação direta. As opções terapêuticas vão desde a colagem de fragmento (que consiste na simples colagem do fragmento com o remanescente do dente), uso de coroa de acetato ou tira de matriz plástica (serve de molde para reconstruir o elemento dentário) e, por fim, a restauração com resina composta direta com auxílio da guia de silicone (Andrade et al., 2009).

A restauração adesiva direta, além de preservar a estrutura dental e exigir menor tempo de tratamento, ganhou espaço com a evolução das propriedades de translucidez, matiz, croma e valor, que são muito similares às do dente a ser restaurado (Pucci et al., 2009). Deve-se considerar que o esmalte é uma estrutura altamente mineralizada composta por prisma e pouca quantidade de água em sua composição, refletindo melhor a luz, enquanto a dentina é uma estrutura tubular orgânica, menos mineralizada e com maior quantidade de água, fazendo com que a interação com a luz seja diferente (Glendor, 2009).

As resinas compostas nanoparticuladas otimizam a adesão dos materiais restauradores aos tecidos do dente, aumentando assim a forma mecânica, adaptação marginal e longevidade das restaurações (Andrade et al., 2009; Bispo, 2010). Desta forma, a resistência mecânica é melhorada, tornando-se semelhante às resinas microhíbridas, somadas às vantagens estéticas de polimento e brilho das resinas micropraticuladas, o que permite realizar um tratamento mais eficaz (Andrade et al., 2009).

No entanto, mesmo com todos os benefícios agregados das resinas compostas, nota-se que há características desfavoráveis que prejudicam o tratamento clínico, como fraturas, microinfiltração marginal e baixa longevidade (Pucci et al., 2009).

Dentre as formas de restaurações, a técnica da guia de silicone (mock-up) é um tratamento que permite a simulação do planejamento a ser realizado, tendo como vantagens o menor risco biológico, estético e funcional (Silva et al., 2012). A utilização desta ferramenta de auxílio juntamente à resina composta contribui para recompor o sorriso de forma estética, funcional e, sobretudo, conservadora, por seguir critérios específicos de alinhamento, proporção e cor, minimizando as necessidades de desgaste dental.
O objetivo desse trabalho foi apresentar um caso clínico de reconstrução de uma fratura coronária do elemento 11 por meio de restauração direta em resina composta nanoparticulada com auxílio de guia de silicone.

\section{CASO CLÍNICO}

Paciente L.B., 13 anos, gênero feminino compareceu à Clínica Integrada do Instituto do Amazonas de Ensino Superior - IAES com trauma dentário do elemento 11. Na anamnese, a paciente relatou insatisfação estética devido a um trauma ocorrido na infância a aproximadamente 5 anos. No exame clínico extraoral não foram verificadas alterações dignas de nota. Entretanto, no exame intraoral observou-se no incisivo central superior direito uma fratura coronária no terço médio sem exposição pulpar e ausência de invasão do espaço biológico (Fig. 1). Radiograficamente, não foi observada alteração periapical aparente. Optou-se, então, pela realização da técnica restauradora direta em resina composta nanoparticulada com auxílio da guia de silicone.

Inicialmente obteve-se a moldagem do paciente com material de impressão alginato (Jeltrate Dentsply) e confecção do modelo de estudo em gesso especial (Durone - Dentsply). Na sequência, realizou-se o enceramento diagnóstico a fim de observar e definir a forma e contorno final da restauração, atendendo aos anseios do paciente. Confeccionou-se então o guia restaurador com silicone de condensação pesada (Clonage - DFL), a partir do modelo de estudo. Desta forma, criou-se a referência para reconstrução considerando o tamanho, largura e harmonia com os dentes adjacentes.

O protocolo clínico iniciou-se com a profilaxia utilizando pedra pomes (Extra fina - Asfer) e água com o auxílio da escova de Robinson (Flat Prophy Brush - RA - White - Microdont) em

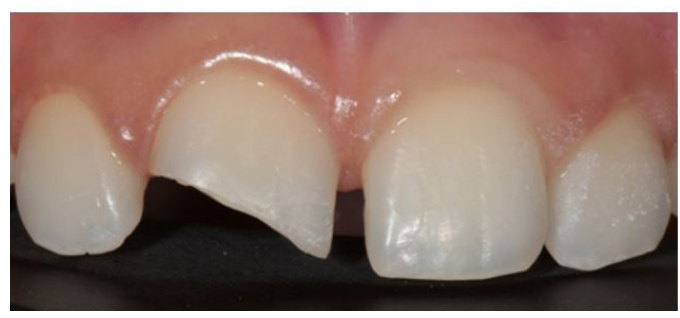

Figura 1 - Caso inicial. Fratura no terço médio do 11 . 
baixa rotação com intuito de realizar uma limpeza superficial do esmalte. A seleção da cor foi baseada na região cervical e incisal do elemento 21 (Fig. 2). As resinas compostas nanoparticuladas escolhidas foram EA2 para esmalte, DA2 para dentina. Além disso, utilizou-se uma resina composta de efeito translúcido, AT, para reproduzir o halo incisal, e uma de valor alto, agregando mais brilho à restauração. Todas as resinas compostas usadas foram da marca Z350 XT (3M/ESPE).

Logo após foi feito o isolamento absoluto da arcada superior para obter o campo operatório limpo e livre de umidade. Com o intuito de mascarar a união dente/restauração e aumentar os valores de adesão, optou-se pela realização de um bisel $(0,5 \mathrm{~mm})$ na face vestibular com angulação de $45^{\circ}$ (Fig. 3).

O remanescente foi condicionado com ácido fosfórico 37\% (Attaque Gel 37\% - Biodinâmica), 15 segundos em dentina e 30 segundos em esmalte, seguido de lavagem abundante e secagem com bolinha de algodão. Criando uma descalcificação seletiva formando poros que, na superfície do esmalte, aumentam o embricamento micromecânico.

O sistema adesivo (Adper ${ }^{\mathrm{TM}}$ Single Bond Plus - 3M/ESPE) foi aplicado com auxílio de um aplicador, seguido de leves jatos de ar para a evaporação do solvente e fotopolimerizado de acordo com as recomendações do fabricante.

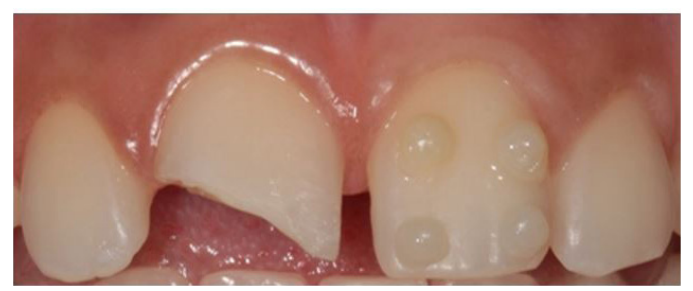

Figura 2 - Seleção de cor (A2 Esmalte / A2 Dentina)

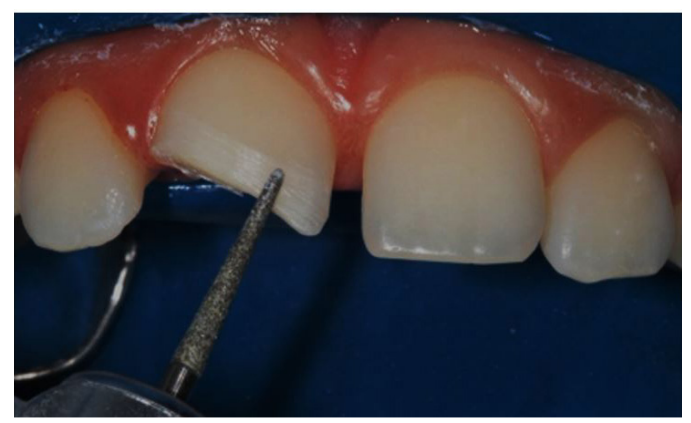

Figura 3 - Confecção do bisel na face vestibular
Os dentes adjacentes foram protegidos com politetrafluoretileno.

Em seguida, a guia de silicone foi posicionada para avaliar a adaptação nos dentes a serem restaurados para confecção adequada da borda incisal e marginal. Seguidamente, um incremento de resina composta cor A2E foi inserido para reconstituir o esmalte palatino (Fig. 4), uma segunda camada na cor A2D foi inserida para reconstituir a dentina artificial e os mamelos dentinários (Fig. 5).

Como a paciente apresentava bordo translúcido, uma resina composta de efeito foi utilizada para conferir aspecto natural ao procedimento restaurador (Fig. 6).

Para restituir o esmalte vestibular, foi inserida uma camada de resina composta na cor $\mathrm{A} 2 \mathrm{E}$ (Fig. 7) e sobre toda a restauração foi utilizada uma resina composta de valor alto.

Para a inserção dos incrementos em resina composta, foi utilizada espátula de inserção $n^{\circ} 1 / 2$ Suprafill Cyann (SS/WHITE) e pincéis de cerdas naturais, permitindo uma superfície mais lisa e polida, possibilitando uma excelente

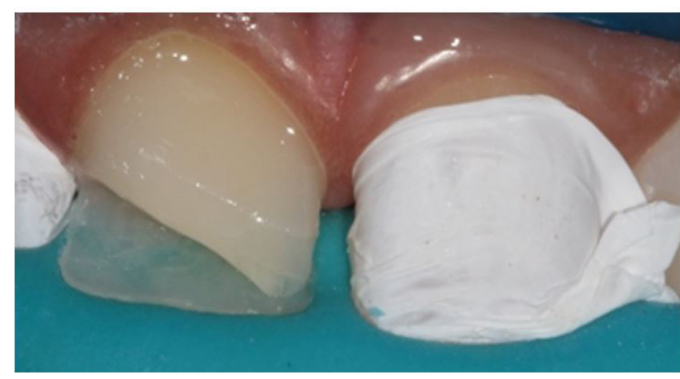

Figura 4 - Aplicação da resina composta referente ao esmalte palatino EA2 (Z350XT - 3M/ESPE).

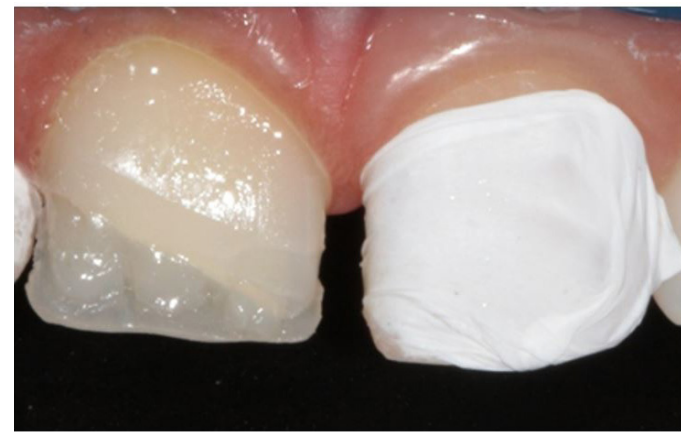

Figura 5 - Aplicação de resina composta para dentina com construção dos lobos dentinários DA2 (Z350XT - 3M/ESPE). 
biocompatibilidade com o tecido gengival na porção cervical da restauração.

Após a remoção do isolamento absoluto, foi feito o ajuste oclusal com o papel carbono Accufilm (Parkell) com movimentos látero-protrusivos e em oclusão cêntrica, para determinar a estabilização dentária, evitando sequelas por trauma oclusal.

Um acabamento grosseiro foi realizado com tiras de lixa de poliéster com movimentos em "S" nas faces interproximais para regularização das bordas proximais e também foi utilizada uma lâmina de bisturi $n^{0} 12$ para remoção dos excedentes cervicais.

Após 24 horas da finalização do procedimento restaurador, realizou-se a etapa de acabamento intermediário, fazendo uso de pontas diamantadas (KG Sorensen $\left.{ }^{\circledR}\right)$ das séries F e FF em alta rotação para realização de ranhuras e depressões dos terços médio e incisal, a fim de criar a textura semelhante ao dente adjacente (Fig. 8). Os discos de lixa (Diamond Pro - $\mathrm{FGM}^{\circledR}$ ) foram empregados

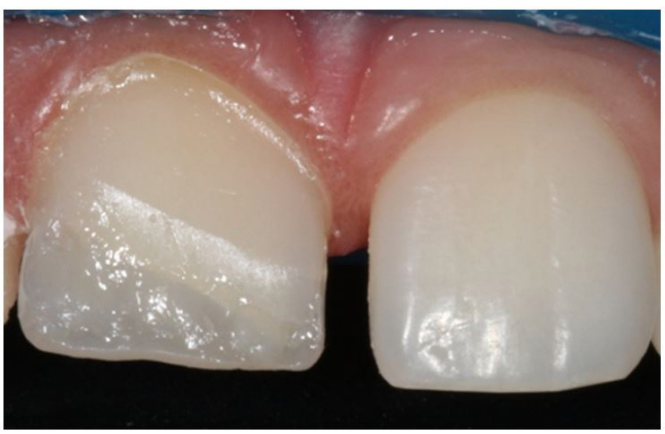

Figura 6 - Entre os lobos, note-se aplicação de resina composta de efeito incisal cinza CT (Z350XT - 3M/ESPE).

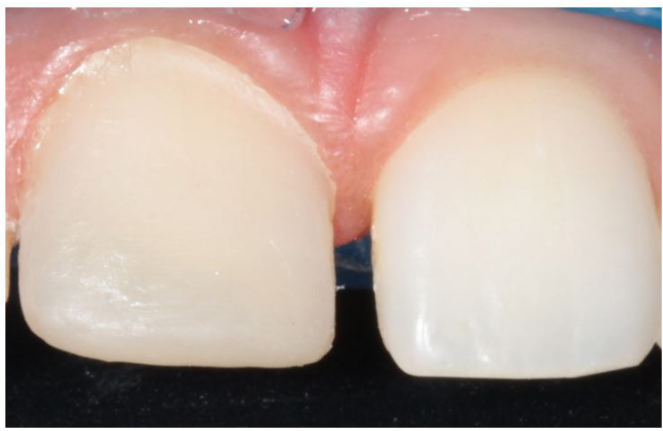

Figura 7 - Camada final de esmalte vestibular EA1 (Z350XT - 3M/ESPE). para a determinação do formato básico do dente em ordem decrescente de granulação, em baixa rotação. Adicionalmente, foram utilizadas pontas de acabamento de borracha (Enhance ${ }^{\circledR}$ ) para resina composta em baixa rotação, que retocaram a anatomia e fizeram o desgaste necessário para uma superfície lisa, uniforme e brilhante.

Logo depois, na etapa do polimento da restauração, objetivando a textura e o brilho da superfície do esmalte, utilizaram-se de discos de feltro seco (Diamond - FGM ${ }^{\circledR}$ ) e pasta diamantada (Diamond Excel - FGM ${ }^{\circledR}$ ) (Fig. 9).

Ao final do procedimento restaurador obteve-se um aspecto mais estético ao sorriso do paciente, melhorando a autoestima (Fig. 10).

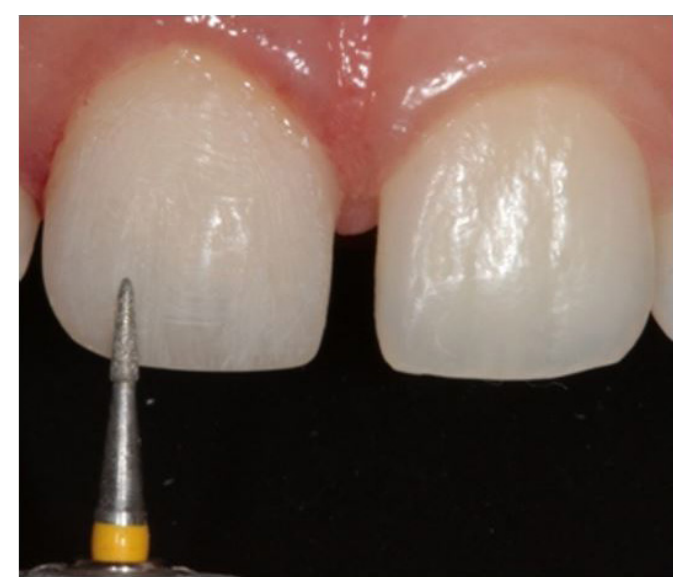

Figura 8 - Reprodução de texturas superficiais e sulcos de desenvolvimentos com ponta diamantada de granulação fina.

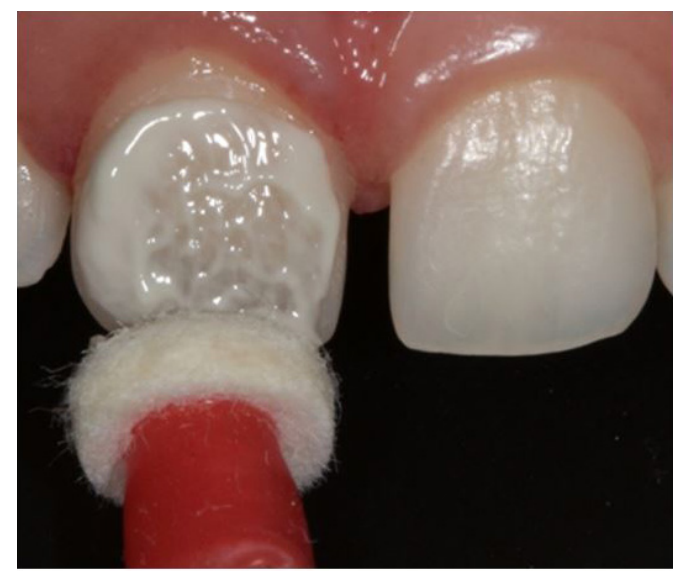

Figura 9 - Polimento obtido com pastas DiamondExcel (FGM) em discos de feltro Diamond Flex (FGM). 


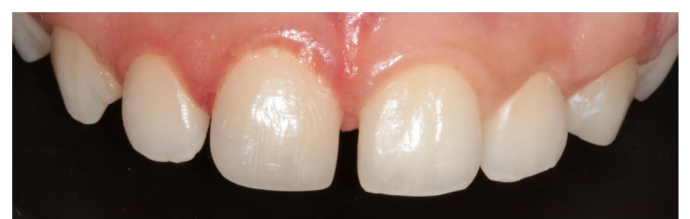

Figura 10 - Resultado final obtido.

\section{DISCUSSÃO}

O presente estudo enfocou o tratamento de uma paciente jovem com fratura dentária em área anterior superior. Nestes casos, o comprometimento da estética exige a escolha de técnica operatória que traga boa relação custo-benefício.

Trata-se de um assunto relevante também por sua alta prevalência. Segundo Gassner et al. (1999), ao avaliar episódios de trauma dental em 6000 pacientes com danos faciais, observou-se que, em $57,8 \%$ das situações, a causa estava relacionada a episódios de acidentes domésticos e brincadeiras, $50,1 \%$ de acidentes esportivos, $38,6 \%$ de acidentes de trabalho, $35,8 \%$ de atos de violência, $34,2 \%$ de acidentes de trânsito e $31 \%$ de acidentes não especificados.

O caso clínico exposto, de fratura coronária no elemento 11, decorreu de um acidente doméstico, tendo sido resina composta nanoparticulada utilizada como mecanismo de reconstrução da estrutura dentária, o que levou a resultados clínicos satisfatórios. A utilização de resina composta se justifica pelos benefícios advindos das propriedades satisfatórias, como excelente polimento e brilho, além da alta resistência à fratura e ao desgaste (Durand et al., 2008).

A desmineralização da dentina e a exposição da rede de fibras colágenas é importante para que haja uma melhor adesão à resina composta. Considerou-se na escolha dos materiais que adesivos de etanol e água apresentam uma maior fixação da resina, além de uma maior durabilidade (Adper ${ }^{\mathrm{TM}}$ Single Bond Plus - 3M/ESPE).

Em se tratando da guia de silicone, Silva et al. (2012) relataram vantagens obtidas com a confecção da muralha de silicone, que nada mais é que um mecanismo de auxílio para o tratamento de restauração dentária, além de servir para reproduzir mais facilmente as referências em tamanho, largura e harmonia com os dentes adjacentes, facilitando assim posterior ajuste estético e funcional. Para Higashi et al. (2006), o uso da matriz de silicone a partir do modelo de estudo permite a visualização prévia e correto posicionamento da estrutura a ser reconstruída. Ambos os autores concluíram que a utilização desta ferramenta auxiliar juntamente à resina composta consegue atingir o objetivo de recompor o sorriso, minimizando as necessidades de desgaste dentário, vindo ao encontro do presente caso, que utilizou esta ferramenta de auxílio.

Segundo Tapia et al. (2012), após a etapa restauradora, faz-se necessário realizar o acabamento da restauração, com a finalidade de reduzir a rugosidade das ranhuras deixadas pelos instrumentos grosseiros de acabamento, resultando em uma superfície lisa e brilhante, pois o mesmo reduz o acúmulo de biofilme.

A técnica utilizada neste caso clínico apresentou resultados estéticos satisfatórios, devolvendo um sorriso mais harmonioso e a completa satisfação da paciente.

\section{CONCLUSÃO}

A técnica da resina composta direta revelou-se uma alternativa viável na reconstrução do dente anterior fraturado, permitindo a manutenção de grande parte da estrutura dentária em uma intervenção clínica realizada em um curto tempo clínico, que garantiu bom custo-benefício à paciente.

\section{REFERÊNCIAS}

Andrade, M. V., Oliveira, L. G. F., Menezes Filho, P. F., \& Silva, C. H. V. (2009). Tendências das resinas composta nanoparticuladas. International Journal of Dentistry, 8(2), 153-157.

Bispo, L. B. (2010). Resina composta nanoparticulada: há superioridade no seu emprego. Revista Dentística, 9(19), 21-24.

Durand, L. B., Monteiro Junior, S., \& Maia, H. P. (2008). Recontorno cosméticos e restaurações adesivas diretas para correção de desarmonias estéticas. Clínica International Journal of Brazilian Dentistry, 2(14), 154-162.

Gassner, R., Bösch, R., Tuli, T., \& Emshoff, R. (1999). Prevalence of dental trauma in 600 patients with facial injuries: implications for prevention. Oral Surgery, Oral Medicine, Oral Pathology, Oral Radiology, and Endodontics, 87(1), 27-33.

Glendor, U. (2008). Epidemiology of traumatic dental injuries: a 12 year review of the literature. Dental Traumatology, 24, 603-611.

Higashi, C., Gomes, J. C., Kina, S., Andrade, O. S., \& Hirata, R. Planejamento estético em 
dentes anteriores. In A. T. Mello, \& E. Miyashita (Eds.), Odontologia estética: planejamento e técnica (Cap. 7). São Paulo: Artes Médicas; 2006. Recuperado em 15 de novembro de 2014, de http://www.ronaldohirata.com.br/data/articles/ planej_estet_dentes_anter_final.pdf

Pucci, C. R., Barcellos, D. C., Batista, G. R., Torres, C. R. G., Borges, A. B., Araújo, M. A. M., \& Gonçalves, S. E. P. (2009). Avaliação longitudinal da microinfiltração utilizando sistemas adesivos convencionais e autocondicionante. La Revue Odontologique, 17(34), 69-75.
Tapia, L. R., Amaral, F. L. B., França, F. M. G., Flório, F. M., Rodrigues, J. A., \& Basting, R. T. (2012). Rugosidade de resinas compostas submetidas a diferentes métodos de acabamento e polimento. Revista de Odontologia da UNESP, 41(4), 254-259.

Silva, R. G., Waechter, D. M., Martins, L. R. M., Barreto, B. C. F., \& Soares, C. J. (2012). Técnicas restauradoras para fraturas coronárias de dentes anteriores traumatizados. UNOPAR Cientifica: Ciências Biológicas e da Saúde, 14(4), 251-256. 